\title{
Distance estimation of howling golden jackals (Canis aureus) using relative sound level
}

\author{
Lukas Graf ${ }^{1}$ (D) . Jennifer Hatlauf ${ }^{1}$ (i)
}

Received: 23 March 2021 / Accepted: 20 July 2021 / Published online: 21 August 2021

(c) The Author(s) 2021

\begin{abstract}
Golden jackals (Canis aureus) display a complex repertoire of calls, utilized in different communication types (e.g., marking territories, attraction of mating partners). Resident golden jackal groups can successfully be detected by active bioacoustic stimulation, as well as with passive recording devices. For monitoring, basic knowledge of the calls of the focal species and potential restrictions and strengths of the monitoring devices should be considered. We therefore tested possible applications of a low-budget autonomous recording unit for bioacoustic golden jackal monitoring and examined the following research questions: How far can group calls be detected? Can the distance to the recording device be estimated? To answer these questions, we placed 11 AudioMoth recording devices in a linear transect to record live imitated and replayed howls. For the estimation of the number of responding animals, the number of howling individuals was determined based on the maximum number of simultaneously visible fundamental frequencies in a spectrogram. To predict the distance of the playback howls to the recording devices, the relative sound level (RSL) of each call was measured and fitted in linear models. Reliable distance estimations using RSL were possible up to $400 \mathrm{~m}$. Estimated number of responding animals showed a negative relationship with distance. Our results present a baseline for future studies and show that AudioMoths can be a helpful asset in distance estimation of golden jackal packs—-both in passive but also active monitoring.
\end{abstract}

Keywords Canidae $\cdot$ Distance estimation $\cdot$ AudioMoth $\cdot$ Relative sound level $\cdot$ Bioacoustic monitoring $\cdot$ Autonomous recording units

\section{Introduction}

The golden jackal (Canis aureus) is a canid species spreading into central and northern Europe from southern and eastern countries such as Bulgaria, Romania, Serbia, and Hungary and is abundant in countries in the Middle East and Asia (Krofel et al. 2017; Spassov and Acosta-Pankov 2019). One method for monitoring this species' presence is bioacoustic monitoring (Giannatos et al. 2005), which can help to gain information of animal abundance (Marques et al. 2013) and has already been used to study species

Communicated by Dries Kuijper.

Jennifer Hatlauf

jennifer.hatlauf@boku.ac.at

1 Department of Integrative Biology and Biodiversity Research (DIB), Institute of Wildlife Biology and Game Management (IWJ), University of Natural Resources and Life Sciences (BOKU), Vienna, Austria across different taxa (Dawson and Efford 2009; Suter et al. 2017). Golden jackals perform an array of different vocalization types, for example long distance calls for territorial defense, social cohesion, or to find a mating partner (Jhala and Moehlman 2004; Comazzi et al. 2016). Their highly complex and long $(29.9 \mathrm{~s} \pm 3.7 \mathrm{~s})$ vocalization repertoires (Comazzi et al. 2016) could theoretically vary between individual packs and meta-populations (Hennelly et al. 2017). During acoustic monitoring, which is usually done from dusk until dawn, researchers mostly estimate the distance to, and the composition of the group based on the heard acoustic signal emitted by the jackals (Giannatos et al. 2005; Szabó et al. 2009; Hatlauf and Hackländer 2016). Passive bioacoustic monitoring, i.e., placing audio recording units in the field to capture vocalizations of the focal species, is used for researching canid species in general (Suter et al. 2017) and also for golden jackals specifically (Comazzi et al. 2016). For confirmation of presence, a fixed recorder might be especially useful when conditions are sub-optimal for researchers to reach the study area frequently (Marques 
et al. 2013). Precise measurements are necessary to estimate population trends and pack size (Comazzi et al. 2016). Imprecise distance estimation potentially leads to under- or overestimation of density indices due to human bias (Yip et al. 2019).

Scott et al. (1981) as well as Nadeau and Conway (2012) noticed that humans are prone to errors in distance estimations of acoustic signals. The given distance estimations were up to 30 times the true distance. This error in distance estimation is likely to increase with the distance to the sound source. Sebastián-González et al. (2018) and Yip et al. (2019) found that the relative sound level (RSL) presents a more reliable distance estimation, thus removing human subjectivity from distance estimations. The RSL is the relative measured emitted energy of a signal in an audio recording that is measured with specific audio software (Yip et al. 2019). As the RSL-which is typically measured in decibels $(\mathrm{dB})$ - has a predictable relationship with distance it can be an estimator for distance.

Recent studies that focus on distance estimation with autonomous recording units (ARUs) mostly used Song Meter devices by Wildlife Acoustics (Suter et al. 2017; Sebastián-González et al. 2018; Yip et al. 2019). In this study, we applied a low-budget alternative, the AudioMoth devices by Open Acoustic Devices. Hill et al. (2018) showed that AudioMoth devices can successfully detect gunshots on distances of over $1 \mathrm{~km}$. This prompted us to investigate AudioMoths for opportunities or weaknesses specifically within golden jackal monitoring.

For our general aim to evaluate the application of AudioMoth devices in distance estimation for golden jackal monitoring with RSL, the broadcasted playback needs to be at a similar sound pressure level (SPL) as a live golden jackal howl. Therefore, we firstly evaluated the SPL of golden jackal howls and secondly analyzed the maximum possible distance for the detection of golden jackal howls using AudioMoth devices. We hypothesized that golden jackal howls (contrary to gunshots) cannot reliably be detected by AudioMoths over a distance of more than $1 \mathrm{~km}$ and that distance and number estimation becomes less precise with increasing distance. Finally, with the predicted distances of the RSL, we suggest adapted minimum grid sizes of AudioMoth devices, to not only be able to confirm jackal presence but also reliably predict distances.

\section{Methods}

\section{Study design}

We used 11 AudioMoth devices (five devices of version 1.0.0 and six devices of version 1.1.0) in this study. We calibrated microphones using a $1 \mathrm{kHz}$ sinus wave at a known
SPL. To analyze the SPL of golden jackal howls we mounted multiple AudioMoths in an enclosure in combination with a camera trap to evaluate the distance from the howling animal to the deployed AudioMoth. The SPL of a single howl on $1 \mathrm{~m}$ distance from the source was calculated, using the formula $L_{2}=L_{1}-\left|20 * \log \left(\frac{r 1}{r 2}\right)\right| \mathrm{dB}$ with the sound source of known SPL as reference. On the basis of this, we proceeded to calculate the SPL of a group howl using the formula $L_{\varepsilon}=10 * \log _{10}\left(10^{\frac{L 1}{10}}+10^{\frac{L_{2}}{10}}+\cdots+10^{\frac{L n}{10}}\right) \mathrm{dB}$, assuming that the animals howl close to each other and at the same SPL, with $L=$ previously calculated SPL and $\mathrm{r}=$ distance. We calculated the SPL of a group howl with four individuals, as four fundamental frequencies were shown at the same time in the spectrogram the standard howling (used previously in monitoring). We used a sound level meter (Peak Meter PM6708) to calibrate the megaphone in the field trials, according to the calculated SPL.

The study area was located in a lowland rural area in eastern Austria without nearby forests and $121 \mathrm{~m}$ above sea level (see study design in Fig. 1 of the Online Resource). We mounted the devices in a linear transect with approximately $100 \mathrm{~m}$ distance between devices and chose additional 20 positions along the transect to be calling stations (CS). The position of each device and each CS was determined using a global positioning device (GPS) device. We covered the devices in plastic bags and fixed them at a height of approximately $1.3 \mathrm{~m}$ to thin metal poles. The lowest distance from an AudioMoth to CS was $8.81 \mathrm{~m}$; the maximal distance was $1540 \mathrm{~m}$. We programmed the AudioMoths to record at a "high gain" and a "sample rate" of $8 \mathrm{kHz}$. A recorded howl of four individuals was played back using a megaphone, set to the previously calculated SPL. The researcher holding the megaphone was kneeling and holding the megaphone approximately $50 \mathrm{~cm}$ above ground to simulate the height of a golden jackal. We played the group howl once at each CS with a megaphone (Pyle PMP57LIA, $50 \mathrm{~W}$ ), followed by a single howl, imitated by a human (the corresponding author).

\section{Wave data analysis}

\section{Distance estimation with RSL}

We analyzed the data in Raven Pro v. 1.5 (Center for Conservation Bioacoustics 2014), using a 512-point Hamming window spectrogram for visualization. We examined RSL using the "max power" function. For better visualization (and thus, detectability), the recordings were amplified by $12 \mathrm{~dB}$, using the "batch amplifier" function. We marked the smallest area possible around the signal as a selection, following the method described by Yip et al. (2019). Selections 
generated in the amplified sound files were then copied back to the original sound files, keeping the original RSL.

\section{Estimating the number of responding animals}

We estimated number of responding animals by counting the simultaneously visible fundamental frequencies in the spectrogram of each detected group howl.

\section{Statistical analysis}

We transferred the selections into the statistic software R 4.0.4 (R Core Team 2021) using the package "Rraven" (Araya-Salas 2017). We then calculated the distance between each CS and the ARU's using the package "sp" (Pebesma and Bivand 2005). Likewise, the bearing of a vocalization relative to the microphone of the ARU was calculated using the package "geosphere" (Hijmans 2019) and classified in variables "front" and "back" depending on the relative direction of the emitted howl relative to the microphone. We tested the known distance of the vocalizations in the resulting dataset (i.e., the selections generated in Raven Pro 1.5.) (Graf and Hatlauf 2021) for normal distribution using a Shapiro-Wilk normality test. No normal distribution was found, thus we tested for differences between the measured SPL and the two call types ("group howl" and "researcher", directions ("back" and "front") and device versions ("1.0.0" and "1.1.0") utilizing a Mann-Whitney $U$-test. We fitted linear models, using the known distance as the response variable.

\section{Analysis of RSL}

We ranked multiple models using an Akaike Information Criterion corrected for small sampling sizes ( $\mathrm{AIC}_{\mathrm{c}}$ ), only selecting the most parsimonious model for further analysis. To estimate the optimal recording distances and enabling reliable distance predictions, we repeatedly removed the top $5 \%$ of the howls with the longest known distance until the Nagelkerke- $R^{2}$ was higher than $R^{2}=0.8$, using $R^{2}$ as an estimator for model prediction quality, resulting in five subsets of data to our models. We ran a $k$-fold means cross validation $(k=10)$ on each model of each data subset with the lowest $\mathrm{AIC}_{\mathrm{c}}$-score, using the mean absolute error (MAE) as a further estimate of model prediction quality. We calculated the distance estimation error as the value of the predicted distance subtracted by the known distance.

\section{Analysis of number of responding animals}

To test whether the number of responding animals can reliably be estimated in spectrograms over distance, we fitted two linear models (one for the full dataset and one for dataset showing the best considered model after $\mathrm{AIC}_{\mathrm{c}}$-ranking), with the estimated number of responding animals as the response variable and the known distance as the predictor variable.

\section{Results}

The calculated SPL of a single golden jackal howl was $101.8 \mathrm{~dB}$. Using the formula for multiple, coherent emitting sound sources, the determined SPL of four simultaneously howling golden jackals resulted in $107.0 \mathrm{~dB}$. Therefore, the megaphone was calibrated to play the recorded group howl at the closest possible SPL. The measured RSL in Raven Pro showed similar maximal values for the recorded group howl $(112.5 \mathrm{~dB})$ and group howl emitted by the megaphone during the field test $(113.7 \mathrm{~dB})$.

Ten of 11 AudioMoth devices recorded wave files as expected, one device had a corrupted file, thus leaving $n=400$ vocalizations to be detected in the spectrograms. $57.5 \%$ of those vocalizations $\left(n_{\text {total }}=230 ; n_{\text {researcher }}=120\right.$; $n_{\text {group }}=110$ ) were detected in the spectrograms. The longest distance to successfully identify vocalizations by means of visually scanning the spectrogram was $814.57 \mathrm{~m}$ for both call types.

No significant differences between "call types" emitted by the megaphone or the howling researcher and "distance" ( $W=6330.5 ; p=0.593$ ) (see Fig. 2 in Online Resource 1), nor between the different "device versions" and "distance" ( $W=6226 ; p=0.5315$ ) were found. Therefore, both "call types" and "device versions" were therefore included in further analyses and not analyzed separately or excluded. Significant differences between the "directions" and "distance" ( $W=3239 ; p<0.001)$ were found, thus all calls with variable "back" $(n=55)$ (where the microphone was aligned opposite from the calling direction) were left out from further analysis, leaving $n_{\text {howls }}=175$ for further analysis.

For each considered dataset, the $\mathrm{AIC}_{\mathrm{c}}$-score was lowest when the RSL was modeled as a second order polynomial term (all for the $\mathrm{AIC}_{\mathrm{c}}$ model selection considered models can be found in Table 1). For the full dataset model, the $k$-fold means cross validation showed a MAE $=82.57 \mathrm{~m}$ $( \pm 14.37 \mathrm{~m})$. Reducing the maximum distance of the dataset by $5 \%$ per step showed, after removing $20 \%$ of the data, the maximal distance was $400.86 \mathrm{~m}, R^{2}$ was above $80 \%$ $\left(R^{2}=0.803\right)$ and the MAE reduced to $39.02 \mathrm{~m}( \pm 7.41 \mathrm{~m})$. The distance estimation error increased with growing distance to the ARU (Fig. 1) and had a maximum of $-576.32 \mathrm{~m}$ for the full dataset model and $-146.63 \mathrm{~m}$ at the $80 \%$ dataset model.

The estimated number of responding animals showed a significant negative relationship with distance for the full dataset $(-0.004 ; p<0.001)$ as well as for the best considered model after $\mathrm{AIC}_{\mathrm{c}}$ model selection $(-0.012 ; p<0.001)$. 
Table 1 Considered models in $\mathrm{AIC}_{\mathrm{c}}$ model selection. The bold models with the lowest $\mathrm{AIC}_{\mathrm{c}}$-score for each respective dataset were considered for the $k$-fold means cross validation. Models were sorted after their $\mathrm{AIC}_{\mathrm{c}}$-score and their $R^{2}$; thus, the model with lowest $\mathrm{AIC}_{\mathrm{c}}$ is at the top. As another measure of model prediction quality, the mean absolute error (MAE) is listed

\begin{tabular}{|c|c|c|c|c|c|c|}
\hline Dataset & Response & Covariates & $\mathrm{AIC}_{\mathrm{c}}$ & $\Delta \mathrm{AIC}_{\mathrm{c}}$ & $R^{2}$ & MAE \\
\hline \multirow[t]{3}{*}{$80 \%$ model } & \multirow[t]{3}{*}{ Known distance } & $\mathrm{dB}+\mathrm{dB}^{2}$ & 1507.9 & 0.00 & 0.803 & \multirow[t]{3}{*}{$39.02 \mathrm{~m}( \pm 7.41 \mathrm{~m})$} \\
\hline & & $\log (\mathrm{dB})$ & 1532.3 & 24.44 & 0.723 & \\
\hline & & $\mathrm{dB}$ & 1553.5 & 45.57 & 0.762 & \\
\hline \multirow[t]{3}{*}{$85 \%$ model } & \multirow[t]{3}{*}{ Known distance } & $\mathrm{dB}+\mathrm{dB}^{2}$ & 1668.9 & 160.98 & 0.724 & \multirow[t]{3}{*}{$47.13 \mathrm{~m}( \pm 11.86 \mathrm{~m})$} \\
\hline & & $\log (\mathrm{dB})$ & 1682.8 & 174.92 & 0.692 & \\
\hline & & $\mathrm{dB}$ & 1697.7 & 189.79 & 0.660 & \\
\hline \multirow[t]{3}{*}{$90 \%$ model } & \multirow[t]{3}{*}{ Known distance } & $\mathrm{dB}+\mathrm{dB}^{2}$ & 1822.1 & 314.17 & 0.692 & \multirow[t]{3}{*}{$55.10 \mathrm{~m}( \pm 12.36 \mathrm{~m})$} \\
\hline & & $\log (\mathrm{dB})$ & 1838.1 & 330.23 & 0.655 & \\
\hline & & $\mathrm{dB}$ & 1853.31 & 345.23 & 0.620 & \\
\hline \multirow[t]{3}{*}{$95 \%$ model } & \multirow[t]{3}{*}{ Known distance } & $\mathrm{dB}+\mathrm{dB}^{2}$ & 1969.1 & 461.25 & 0.667 & \multirow[t]{3}{*}{$65.52 \mathrm{~m}( \pm 12.55 \mathrm{~m})$} \\
\hline & & $\log (d B)$ & 1989.2 & 481.31 & 0.616 & \\
\hline & & $\mathrm{dB}$ & 2004.9 & 496.98 & 0.576 & \\
\hline \multirow[t]{3}{*}{ Full model } & \multirow[t]{3}{*}{ Known distance } & $\mathrm{dB}+\mathrm{dB}^{2}$ & 2197.6 & 689.71 & 0.566 & \multirow[t]{3}{*}{$82.574 \mathrm{~m}( \pm 14.37 \mathrm{~m})$} \\
\hline & & $\mathrm{dB}$ & 2212.2 & 704.34 & 0.489 & \\
\hline & & $\log (\mathrm{dB})$ & 2224.2 & 716.34 & 0.523 & \\
\hline
\end{tabular}

Fig. 1 Direct relationships between distance estimation error and distance in both datasets; the $x$-axis shows the distance and the $y$-axis the estimation error calculated as the value of the predicted distances subtracted by the known distance. The RSL predicted distance error increases with increasing known distance to the ARU. Left is the plot of the full model $(n=175)$; the right plot is the reduced " $80 \%$ model" $(n=140)$. Points represent plotted raw data. Gray bands indicate confidence bands

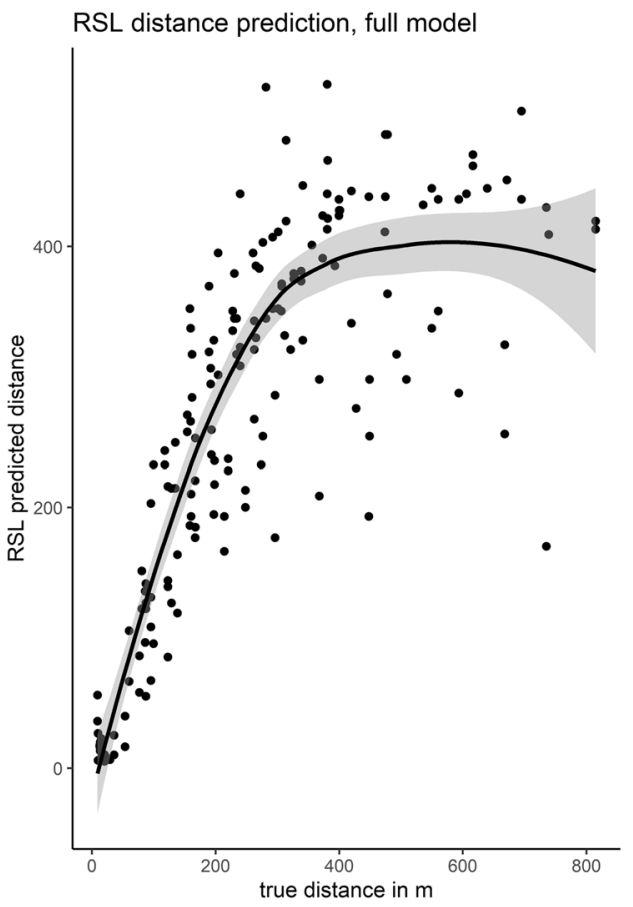

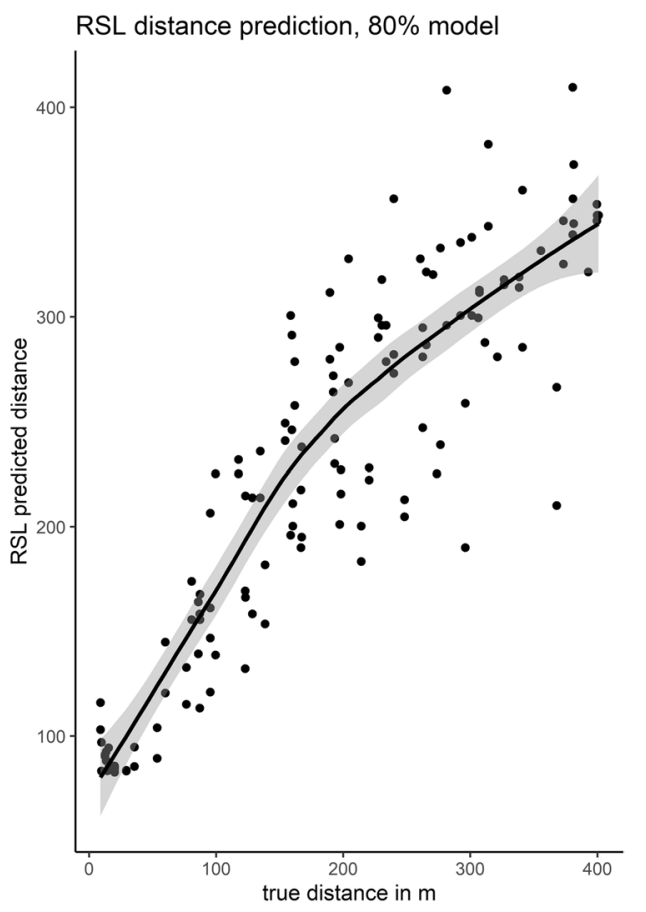

\section{Discussion}

Wave files recorded by AudioMoth devices in our study allowed for visual detection of golden jackal group howls in spectrograms at distances of over $800 \mathrm{~m}$, confirming our first hypothesis, that howls can't be detected at over $1 \mathrm{~km}$ distance. Our best model reliably predicts the distance to the recording AudioMoth up to $400 \mathrm{~m}$. Estimated number of responding animals showed a strong negative relationship with the distance in both considered models, indicating that abundance of golden jackals (at higher distances) might therefore be underestimated in a "realworld" analysis, where the group size of the golden jackal pack is not previously known. Estimation of group size and group composition can be done differently using other passive monitoring applications, like DNA analyses through scats (Hatlauf et al. 2021). Nevertheless, we found that our models reliably estimate distances of howling golden jackal packs. Though occasionally the distance estimations given by our models showed a high MAE, they are likely still more accurate than those given by human researchers (Scott et al. 1981; Nadeau and Conway 2012). 
Future research should emphasize more on this assumption and include direct comparisons between estimations of researchers in the field and known distances and group compositions. However, from personal experience in fieldwork, the distance estimation by researchers is prone to high diversity and often leads to discussions with insecurities and usually with lack of any real confirmations or proof. Learning from our study, it could be an option to implement the (easy portable) recording devices into active bioacoustic monitoring to remove human subjectivity in distance estimation (Yip et al. 2019).

To further improve RSL-based study designs for golden jackal research, it is suggested to study a greater number of howls with known distance to calculate the SPL of a single golden jackal howl and to capture possible plasticity of SPLs. However, Suter et al. (2017) showed the mean SPL of a howling wolf to be $\sim 107.8 \mathrm{~dB}$. Our calculated SPL of a golden jackal howl being $101.8 \mathrm{~dB}$ is within good biological reason. Furthermore, we did not include possible covariates in this study, such as topographical relief, wind situation, vegetation and habitat type or time of day, what could possibly have influenced the model quality (Halfwerk et al. 2011; Maciej et al. 2011; Morrill et al. 2013). Thus, we recommend the training of location specific models, as distance prediction quality might be influenced by surrounding habitats or the time of day. Furthermore, different weather conditions (e.g., strong winds; heavy rain) or insects near the ARU might further alter the RSL. To account for these potentially negative effects, quality thresholds, such as the signal to noise ratio (SNR), can be integrated.

Installing multiple AudioMoths in an area can be an effective tool for bioacoustic monitoring. They are able to capture howling responses that are inaudible for the researchers during active bioacoustic monitoring. Home range size of golden jackals is highly variable and dependent on different factors like prey availability or hiding options. In an area of 2-10 km² (Giannatos et al. 2005; Rotem et al. 2011; Lanszki et al. 2019), a considerable amount of AudioMoth devices would be needed to successfully predict distances of golden jackals using RSL. AudioMoth devices are built with monodirectional microphones and given a $400 \mathrm{~m}$ optimal prediction distance between each device, two devices (if not four, to reduce potential $\mathrm{dB}$ loss on the sides of the devices) per recording station would be needed, to account for the significant underperformance from the "back" of the device. This is reflected in our significant differences between the variables "front" and "back" leading to exclusion of the latter. There are considerable pros and cons for the application of AudioMoths-further studies should test the influence of the signal reaching the AudioMoths laterally to examine the optimal number of devices per recording station. However, installing multiple AudioMoths would again increase the costs and the time spent on analyzing the spectrograms-but could improve overall data quality. Using other autonomous recording units (with a more sensitive microphone) might further increase the recording quality (Suter et al. 2017; Yip et al. 2019) but may also considerably increase the costs per recording station. The long and complex vocalizations of golden jackals (29.9 $\mathrm{s} \pm 3.7 \mathrm{~s}$ ) (Comazzi et al. 2016) on the other hand could impede implementation of recognizer software or codes. Successful implementation of these would further improve the overall accuracy of distance estimation and again reduce working hours (Yip et al. 2019). Installing the AudioMoths in a defined grid would furthermore allow the acoustic triangulation of howling packs, by using the known GPS-positions of the AudioMoths as reference points for triangulation (Kershenbaum et al. 2019; O'Gara et al. 2020).

Our study highlights the possible applications, strengths, and weaknesses of AudioMoths specifically in bioacoustic golden jackal monitoring. We conclude that the AudioMoth recording devices are a helpful addition to improve data quality. Especially when research teams are small or they work in large study areas, deploying multiple AudioMoths might increase overall data quantity. Besides the help within passive acoustic monitoring, an AudioMoth device can even be a simple inclusion during active bioacoustic monitoring for a later confirmation of the estimated distance and removing estimation bias.

Supplementary Information The online version contains supplementary material available at https://doi.org/10.1007/s13364-021-00587-2.

Acknowledgements We are grateful for the help during fieldwork by Matthias Amon. We thank Johannes and Carmen Lukasser, Emanuel Lichtenstein, and Hans Acs for their support in recording golden jackal howls. We are especially thankful for Robin Sandfort for providing background information and help with the first analyses; we furthermore would like to thank Eva Schöll and Fabian Knufinke for valuable comments on methods and the manuscript and two anonymous reviewers for their constructive reviews.

Author contribution LG and JH both designed this study, conducted the fieldwork, and wrote the manuscript.

Funding Open access funding was provided by the University of Natural Resources and Life Sciences Vienna (BOKU). JH was a recipient of the DOC Fellowship of the Austrian Academy of Sciences at the Institute of Wildlife Biology and Game management.

Code and data availability Code and data are available from the repository: 10.17605/OSF.IO/W2V9K.

\section{Declarations}

Consent for publication All authors consent to the publication of this manuscript.

Conflict of interest The authors declare no competing interests. 
Open Access This article is licensed under a Creative Commons Attribution 4.0 International License, which permits use, sharing, adaptation, distribution and reproduction in any medium or format, as long as you give appropriate credit to the original author(s) and the source, provide a link to the Creative Commons licence, and indicate if changes were made. The images or other third party material in this article are included in the article's Creative Commons licence, unless indicated otherwise in a credit line to the material. If material is not included in the article's Creative Commons licence and your intended use is not permitted by statutory regulation or exceeds the permitted use, you will need to obtain permission directly from the copyright holder. To view a copy of this licence, visit http://creativecommons.org/licenses/by/4.0/.

\section{References}

Araya-Salas M (2017) Connecting R and 'Raven' Sound Analysis Software [R package Rraven version 1.0.8]

Center for Conservation Bioacoustics (2014) Raven Pro: interactive sound analysis software (version 1.5). The Cornell Lab of Ornithology, Ithaca. www.ravensoundsoftware.com/

Comazzi C, Mattiello S, Friard O, Filacorda S, Gamba M (2016) Acoustic monitoring of golden jackals in Europe: setting the frame for future analyses. Bioacoustics 25:267-278. https://doi. org/10.1080/09524622.2016.1152564

Dawson DK, Efford MG (2009) Bird population density estimated from acoustic signals. J Appl Ecol 46:1201-1209. https://doi.org/10. 1111/j.1365-2664.2009.01731.x

Giannatos G, Marinos Y, Maragou P, Catsadorakis G (2005) The status of the golden jackal (Canis aureus L.) in Greece. Belg J Zool:145-149

Graf L, Hatlauf J (2021) Data and procedure for: distance estimation of howling golden jackals (Canis aureus) using relative sound level. https://doi.org/10.17605/OSF.IO/W2V9K

Halfwerk W, Bot S, Buikx J, van der Velde M, Komdeur J, ten Cate C, Slabbekoorn H (2011) Low-frequency songs lose their potency in noisy urban conditions. Proc Natl Acad Sci U S A 108:1454914554. https://doi.org/10.1073/pnas. 1109091108

Hatlauf J, Böcker F, Wirk L, Collet S, Schley L, Szabó L, Hackländer K, Heltai M (2021) Jackal in hide: detection dogs show first success in the quest for golden jackal (Canis aureus) scats. Mamm Res:1-10. https://doi.org/10.1007/s13364-020-00537-4

Hatlauf J, Hackländer K (2016) Preliminary results for golden jackal (Canis aureus) survey in Austria. Beiträge zur Jagd- und Wildforschung 41. Gesellschaft für Wildtier- und Jagdforschung e.V. (GWJF), Melsungen:295-306

Hennelly L, Habib B, Root-Gutteridge H, Palacios V, Passilongo D (2017) Howl variation across Himalayan, North African, Indian, and Holarctic wolf clades: tracing divergence in the world's oldest wolf lineages using acoustics. Curr Zool 63:341-348. https://doi. org/10.1093/cz/zox001

Hijmans RJ (2019) Spherical Trigonometry [R package geosphere version 1.5-10]

Hoffmann M, Arnold J, Duckworth JW, Jhala Y, Kamler JF, Krofel M (2018) Canis aureus (errate version published in 2020). The IUCN Red List of Threatened Species 2018

Hill AP, Prince P, Piña Covarrubias E, Doncaster CP, Snaddon JL, Rogers A (2018) AudioMoth: evaluation of a smart open acoustic device for monitoring biodiversity and the environment. Methods Ecol Evol 9:1199-1211. https://doi.org/10.1111/2041-210X. 12955

Jhala Y, Moehlman P (2004) Golden jackal Canis aureus Linnaeus, 1758. In: Sillero-Zubiri C, Hoffmann M, Macdonald DW (eds) Canids: Foxes, Wolves, Jackals and Dogs. IUCN/SSC Canid Specialist Group:156-161
Kershenbaum A, Owens JL, Waller S (2019) Tracking cryptic animals using acoustic multilateration: a system for long-range wolf detection. J Acoust Soc Am 145:1619. https://doi.org/10.1121/1. 5092973

Krofel M, Giannatos G, Ćirovič D, Stoyanov S, Newsome T (2017) Golden jackal expansion in Europe: a case of mesopredator release triggered by continent-wide wolf persecution?Hystrix Ital J Mammal 28.https://doi.org/10.4404/hystrix-28.1-11819

Lanszki J, Heltai M, Kövér G, Zalewski A (2019) Non-linear relationship between body size of terrestrial carnivores and their trophic niche breadth and overlap. Basic Appl Ecol 38:36-46. https://doi. org/10.1016/j.baae.2019.06.004

Maciej P, Fischer J, Hammerschmidt K (2011) Transmission characteristics of primate vocalizations: implications for acoustic analyses. PLoS ONE 6:e23015. https://doi.org/10.1371/journal.pone.00230 15

Marques TA, Thomas L, Martin SW, Mellinger DK, Ward JA, Moretti DJ, Harris D, Tyack PL (2013) Estimating animal population density using passive acoustics. Biol Rev Camb Philos Soc 88:287309. https://doi.org/10.1111/brv.12001

Morrill RJ, Thomas AW, Schiel N, Souto A, Miller CT (2013) The effect of habitat acoustics on common marmoset vocal signal transmission. Am J Primatol 75:904-916. https://doi.org/10.1002/ ajp. 22152

Nadeau CP, Conway CJ (2012) Field evaluation of distance-estimation error during wetland-dependent bird surveys. Wildl Res 39:311. https://doi.org/10.1071/WR11161

O'Gara JR, Wieder CA, Mallinger EC, Simon AN, Wydeven AP, Olson ER (2020) Efficacy of acoustic triangulation for gray wolves. Wildl Soc Bull 81:535. https://doi.org/10.1002/wsb.1089

Pebesma EJ, Bivand RS (2005) Classes and methods for spatial data in R. R News 5:9-13

R Core Team (2021) R: a language and environment for statistical computing. R Foundation for Statistical Computing, Vienna. http:// www.R-project.org/

Rotem G, Berger H, King R, Kutiel PB, Saltz D (2011) The effect of anthropogenic resources on the space-use patterns of golden jackals. J Wildl Manag 75:132-136. https://doi.org/10.1002/jwmg.9

Scott JM, Ramsey FL, Kepler BC (1981) Distance estimation as a variable in estimating bird numbers from vocalizations. Stud Avian Biol:333-340

Sebastián-González E, Camp RJ, Tanimoto AM, de Oliveira PM, Lima BB, Marques TA, Hart PJ (2018)Density estimation of soundproducing terrestrial animals using single automatic acoustic recorders and distance sampling.ACE 13.https://doi.org/10.5751/ ACE-01224-130207

Spassov N, Acosta-Pankov I (2019) Dispersal history of the golden jackal (Canis aureus moreoticus Geoffroy, 1835) in Europe and possible causes of its recent population explosion. Biodivers Data J 7:e34825. https://doi.org/10.3897/BDJ.7.e34825

Suter SM, Giordano M, Nietlispach S, Apollonio M, Passilongo D (2017) Non-invasive acoustic detection of wolves. Bioacoustics 26:237-248. https://doi.org/10.1080/09524622.2016.1260052

Szabó L, Heltai M, Szúcs E, Lanszki J, Lehoczki R (2009) Expansion range of the golden jackal in Hungary between 1997 and 2006. Mammalia 73. https://doi.org/10.1515/MAMM.2009.048

Yip DA, Knight EC, Haave-Audet E, Wilson SJ, Charchuk C, Scott CD, Sólymos P, Bayne EM (2019) Sound level measurements from audio recordings provide objective distance estimates for distance sampling wildlife populations. Remote Sens Ecol Conserv 71(27):59. https://doi.org/10.1002/rse2.118

Publisher's note Springer Nature remains neutral with regard to jurisdictional claims in published maps and institutional affiliations. 